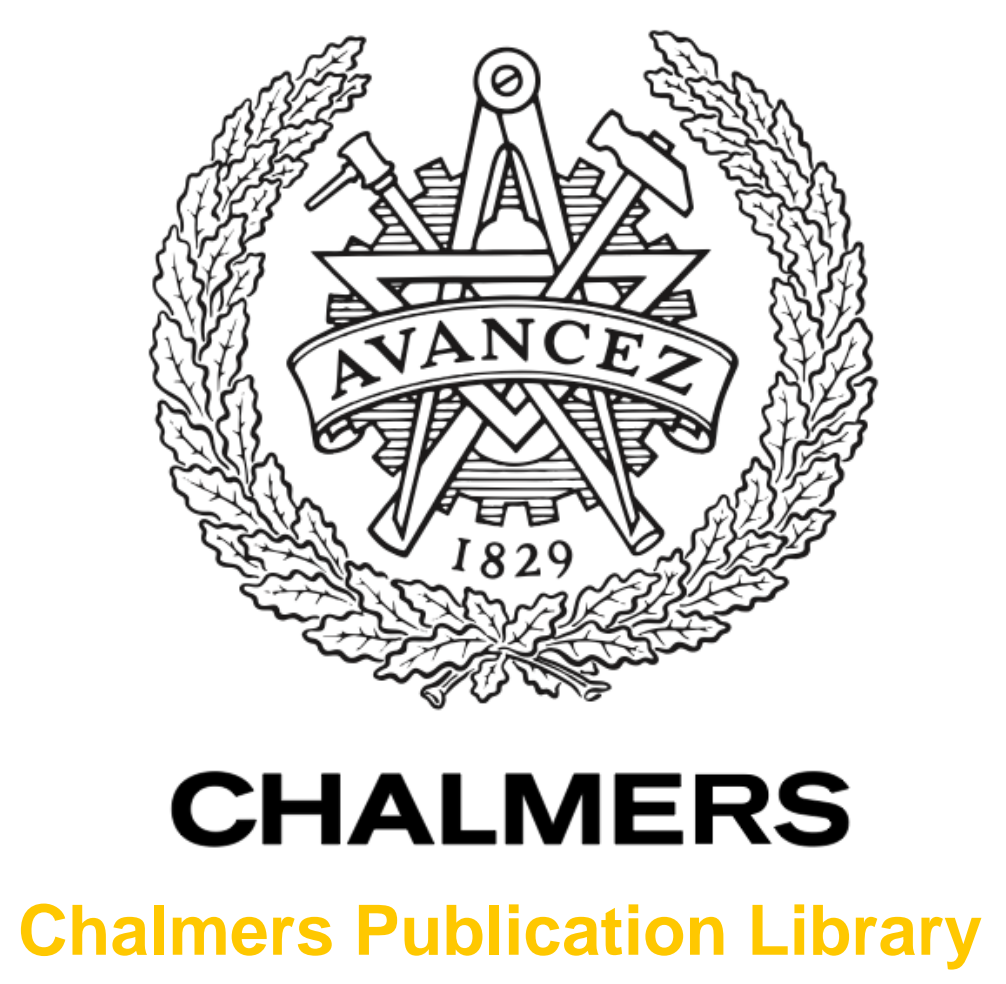

Electromagnetic Source Modeling using Phase Retrieval Methods

This document has been downloaded from Chalmers Publication Library (CPL). It is the author's version of a work that was accepted for publication in:

30th URSI General Assembly and Scientific Symposium, URSIGASS 2011, Istanbul, 13-20 August 2011

Citation for the published paper:

Johansson, M. ; Lui, H. ; Fhager, A. (2011) "Electromagnetic Source Modeling using Phase Retrieval Methods". 30th URSI General Assembly and Scientific Symposium, URSIGASS 2011, Istanbul, 13-20 August 2011 pp. 1-2.

http://dx.doi.org/10.1109/URSIGASS.2011.605131

Down厂oaded from: http://publications.lib.chalmers.se/publication/150000

Notice: Changes introduced as a result of publishing processes such as copy-editing and formatting may not be reflected in this document. For a definitive version of this work, please refer to the published source. Please note that access to the published version might require a subscription. 


\title{
Electromagnetic source modeling using phase retrieval methods
}

\author{
Markus Johansson, Hoi-Shun Lui, Andreas Fhager and Mikael Persson \\ Chalmers University of Technology, SE-41296, Gothenburg Sweden, markusjo@chalmers.se
}

\begin{abstract}
Modeling of the field distributions from electromagnetic sources is of interest for various applications for example electromagnetic compatibility investigations, near-field to far-field transformations, antenna diagnostics and electromagnetic dosimetry. In order to determine whether exposure safety guidelines, such as the EU directive 2004/40/EC, are complied with, source modeling methods are important.

Two methods for determining the total field, including phase information, when only field amplitudes have been measured on a set of planes in front of an electromagnetic source have been developed. The first method, the adjoint field method, is a gradient based optimization algorithm based on the adjoint fields. The second method, the phase angle gradient method, employs an optimization algorithm based on the phase angle gradients of a functional.

Promising results have been obtained both for numerical test cases and for measured field.

\section{The adjoint field method}

The aim of the adjoint field method is to retrieve the phase of the field in front of an electromagnetic source using measured field amplitudes. In the adjoint field method measured field amplitudes on 3 parallel measurement planes in front of an electromagnetic source are used to calculate the phase angles. The measured amplitudes on the measurement plane closest to the source, plane 1, are used as a source, with the field in all points on the plane set to be in phase. Moreover equivalent dielectric properties between the two measurement planes that are closest to the electromagnetic source, are searched for. These properties should together with the source on plane 1 give numerically calculated correct field, amplitude as well as phase, on the other planes. A gradient-based optimization algorithm, that minimize the difference between calculated and measured field, is used to search for the equivalent dielectric properties. The finitedifference time-domain (FDTD) method is used for the field calculations in the algorithm.
\end{abstract}

\section{The phase angle gradient method}

The goal for the phase angle gradient method (PAGM) is to retrieve the phase of the field from an electromagnetic source, using measured field amplitudes on 3 parallel planes in front of the source. Unlike the adjoint field method, the PAGM searches for the correct phase angles on the plane closest to the source, plane 1, directly. The PAGM is gradientbased and searches for phase angles on plane 1, that minimize the difference between calculated and measured field amplitudes on the other planes.

According to the field equivalence principle the electromagnetic source can be represented with an equivalent magnetic surface current density, that can be calculated using the field on plane 1 . The equivalent magnetic current density and the calculated field it gives on the other measurement planes, can be regarded as functions of the unknown phase angles of the field on plane 1 , since the field amplitudes are known on plane 1.

After the phase angles on plane 1 have been initiated, for example to be zero, the resulting field estimates on the other planes can be calculated. To find the correct phase, the initial angles are altered in small steps, so that the calculated field amplitudes converge to the measured field. A functional that is a measure of the difference between calculated and measured field amplitudes have been defined. The phase angles are changed in the opposite direction of the phase angle gradients of the functional, so that it is minimized. 


\section{Results for numerical test cases}

The adjoint field method and the PAGM have been compared. The methods were tested with $500 \mathrm{MHz}$ field from an infinitesimal dipole. It was found that the PAGM performed better than the adjoint field method. Moreover it gave phase angles that are in excellent agreement with the correct field.

A performance evaluation of the PAGM has also been performed. Phase retrieval based on field values for different measurement plane sizes and separations between the planes have been studied. Moreover the method have been tested for different initial phase distributions. The results show that the phase retrieval works well, even if the separation between the measurement planes is small in terms of wavelengths.

\section{Results for measured field}

The PAGM have also been tested for magnetic flux density with the frequency $50 \mathrm{~Hz}$. The method was tested with measured field amplitudes in front of a transformer. A robot was used to move a measurement probe between the measurement points in front of the transformer.

Field amplitudes on 3 measurement planes were used to calculate the phase. An extra plane further away from the source was used for validation purposes. If the obtained phase angles are similar to the correct ones, they should give calculated field amplitudes that are similar to the measured ones. The calculated field amplitudes, that the retrieved phase gave, on the extra measurement plane agreed well with measured amplitudes.

\section{Conclusions}

The phase angle gradient method worked well both for the numerical test cases and for the case with measured field values in front of a transformer. The method is useful for modeling of electromagnetic sources and have the important advantage that it can be used for many different sources without detailed knowledge of the sources. 